DESY 00-051

TUM-HEP-367/00

hep-ph/0003323

March 2000

\title{
Constraints on the CKM Angle $\gamma$ and Strong Phases from $B \rightarrow \pi K$ Decays
}

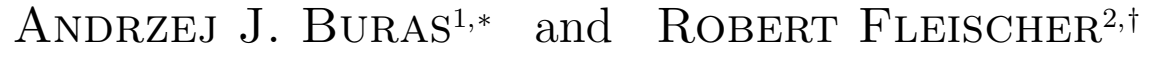 \\ ${ }^{1}$ Technische Universität München, Physik Department, D-85748 Garching, Germany \\ ${ }^{2}$ Deutsches Elektronen-Synchrotron DESY, Notkestr. 85, D-22607 Hamburg, Germany
}

\begin{abstract}
As we pointed out recently, the neutral decays $B_{d} \rightarrow \pi^{\mp} K^{ \pm}$and $B_{d} \rightarrow \pi^{0} K$ may provide non-trivial bounds on the CKM angle $\gamma$. In this paper, we reconsider this approach in the light of recent CLEO data, which look very interesting. In particular, the results for the corresponding CP-averaged branching ratios are in favour of strong constraints on $\gamma$, where the second quadrant is preferred. Such a situation would be in conflict with the standard analysis of the unitarity triangle. Moreover, constraints on a CP-conserving strong phase $\delta_{\mathrm{n}}$ are in favour of a negative value of $\cos \delta_{\mathrm{n}}$, which would be in conflict with the factorization expectation. In addition, there seems to be an interesting discrepancy with the bounds that are implied by the charged $B \rightarrow \pi K$ system: whereas these decays favour a range for $\gamma$ that is similar to that of the neutral modes, they point towards a positive value of $\cos \delta_{\mathrm{c}}$, which would be in conflict with the expectation of equal signs for $\cos \delta_{\mathrm{n}}$ and $\cos \delta_{\mathrm{c}}$. If future data should confirm this "puzzle", it may be an indication for new-physics contributions to the electroweak penguin sector, or a manifestation of large non-factorizable $S U(3)$-breaking effects.
\end{abstract}

\footnotetext{
*E-mail: Andrzej.Buras@feynman.t30.physik.tu-muenchen.de

${ }^{\dagger}$ E-mail: Robert.Fleischer@desy.de
} 



\section{Introduction}

In order to probe the angle $\gamma$ of the unitarity triangle of the Cabibbo-Kobayashi-Maskawa (CKM) matrix at the $B$-factories, $B \rightarrow \pi K$ decays play an outstanding role. Remarkably, already $\mathrm{CP}$-averaged branching ratios of such channels may imply very non-trivial constraints on $\gamma$. So far, the studies of these bounds have focussed on the following two systems: $B_{d} \rightarrow$ $\pi^{\mp} K^{ \pm}, B^{ \pm} \rightarrow \pi^{ \pm} K[\mathbb{1}]$, and $B^{ \pm} \rightarrow \pi^{0} K^{ \pm}, B^{ \pm} \rightarrow \pi^{ \pm} K[\mathbb{Z}]$; they have received a lot of attention in the literature. In a recent paper [3], we pointed out that also the neutral decays $B_{d} \rightarrow \pi^{\mp} K^{ \pm}$ and $B_{d} \rightarrow \pi^{0} K$ may be interesting in this respect, and presented a general formalism, allowing us to describe all three $B \rightarrow \pi K$ systems within the same theoretical framework. Since the CLEO collaboration has reported the observation of the $B_{d} \rightarrow \pi^{0} K$ channel in the summer of 1999, which finalizes the search for all four $B \rightarrow \pi K$ modes, we have reanalysed our approach in view of these new data. It turns out that the new CLEO results [4 favour interesting bounds on $\gamma$ from the neutral $B \rightarrow \pi K$ decays. Here the key quantities are the following ratios of $\mathrm{CP}$-averaged branching ratios [3]:

$$
\begin{aligned}
R & \equiv \frac{\mathrm{BR}\left(B_{d}^{0} \rightarrow \pi^{-} K^{+}\right)+\mathrm{BR}\left(\overline{B_{d}^{0}} \rightarrow \pi^{+} K^{-}\right)}{\operatorname{BR}\left(B^{+} \rightarrow \pi^{+} K^{0}\right)+\mathrm{BR}\left(B^{-} \rightarrow \pi^{-} \overline{K^{0}}\right)}=0.95 \pm 0.28 \\
R_{\mathrm{c}} & \equiv 2\left[\frac{\mathrm{BR}\left(B^{+} \rightarrow \pi^{0} K^{+}\right)+\mathrm{BR}\left(B^{-} \rightarrow \pi^{0} K^{-}\right)}{\operatorname{BR}\left(B^{+} \rightarrow \pi^{+} K^{0}\right)+\mathrm{BR}\left(B^{-} \rightarrow \pi^{-} \overline{K^{0}}\right)}\right]=1.27 \pm 0.47 \\
R_{\mathrm{n}} & \equiv \frac{1}{2}\left[\frac{\mathrm{BR}\left(B_{d}^{0} \rightarrow \pi^{-} K^{+}\right)+\mathrm{BR}\left(\overline{B_{d}^{0}} \rightarrow \pi^{+} K^{-}\right)}{\mathrm{BR}\left(B_{d}^{0} \rightarrow \pi^{0} K^{0}\right)+\mathrm{BR}\left(\overline{B_{d}^{0}} \rightarrow \pi^{0} \overline{K^{0}}\right)}\right]=0.59 \pm 0.27,
\end{aligned}
$$

where the factors of 2 and $1 / 2$ have been introduced to absorb the $\sqrt{2}$ factors originating from the wavefunctions of the neutral pions; the errors of the experimental results given in Ref. [4] have been added in quadrature. If these ratios are found to be smaller than one, they can be converted directly into constraints on $\gamma$ without any additional information. When the $B_{d} \rightarrow \pi^{\mp} K^{ \pm}, B^{ \pm} \rightarrow \pi^{ \pm} K$ channels were observed in 1997 by the CLEO collaboration, the first results gave $R=0.65 \pm 0.40$, and the bound on $\gamma$ presented in Ref. [四] led to great excitement in the $B$-physics community. In the case of $R_{\mathrm{n}}$, we now face a similarly exciting possibility, which we will discuss in more detail in this paper. However, in comparison with the original bound derived in [1], the neutral strategy has certain theoretical advantages, connected mainly with the impact of rescattering processes [5]-[7] and electroweak penguin topologies.

If one of the ratios $R_{(\mathrm{c}, \mathrm{n})}$ specified in (1)-(3) is found to be larger than one, additional experimental information is required to constrain $\gamma$. To this end, we have then to fix sloppily speaking - certain ratios of "tree" to "penguin" amplitudes. Such an input allows us also to obtain stronger constraints on $\gamma$ in the case of $R_{(\mathrm{c}, \mathrm{n})}<1$. The least fortunate case for the bounds on $\gamma$ would be $R_{(\mathrm{c}, \mathrm{n})}$ close to 1 . If CP-violating asymmetries in the channels appearing in the numerators in (1)-(3) can be measured, it is possible to go beyond the bounds on $\gamma$ and to determine this angle, also in the case of $R_{(\mathrm{c}, \mathrm{n})}=1$. A first analysis of such CP asymmetries has recently been performed by the CLEO collaboration [8], where all results are unfortunately still consistent with zero. It is also possible to obtain theoretical upper bounds on such $\mathrm{CP}$ asymmetries. For instance, the ratio of the measured CP-averaged $B_{d} \rightarrow \pi^{+} \pi^{-}$ and $B_{d} \rightarrow \pi^{\mp} K^{ \pm}$branching ratios implies $\left|\mathcal{A}_{\mathrm{CP}}^{\mathrm{dir}}\left(B_{d} \rightarrow \pi^{\mp} K^{ \pm}\right)\right| \lesssim 0.3[9]$. 
It is an interesting feature of the bounds on $\gamma$ that they prefer values in the second quadrant, which would be in conflict with the standard analysis of the unitarity triangle [10. Other arguments for $\cos \gamma<0$ using $B \rightarrow P P, P V$ and $V V$ decays were recently given in [12 (see also [9]). We would like to point out that, in addition to the bounds on $\gamma$, one may also derive constraints on CP-conserving strong phases $\delta_{\mathrm{n}}$ and $\delta_{\mathrm{c}}$ from the neutral and charged $B \rightarrow \pi K$ decays, respectively. Whereas the present CLEO data favour a positive value of $\cos \delta_{\mathrm{c}}$, as is expected in the factorization approximation, they point towards a negative value of $\cos \delta_{\mathrm{n}}$. However, on the basis of simple dynamical considerations, one would expect that $\delta_{\mathrm{n}}$ and $\delta_{\mathrm{c}}$ do not differ dramatically from each other. The present data do of course not allow us to draw any definite conclusions. However, if the future data should confirm this interesting "puzzle", it may be an indication for new-physics contributions to the electroweak penguin sector, or a manifestation of large non-factorizable $S U(3)$-breaking effects.

The outline of this paper is as follows: in Section 2, we repeat briefly the general formalism developed in [3]. The bounds on $\gamma$ are discussed in view of the recent CLEO data in Section 3, where we also have a brief look at constraints in the $\bar{\varrho}-\bar{\eta}$ plane of the Wolfenstein parameters [13], generalized as in Ref. [14]. In Section 4, we turn to the constraints on the strong phases $\delta_{\mathrm{n}}$ and $\delta_{\mathrm{c}}$. Finally, a few concluding remarks are given in Section 5 .

\section{General Formalism}

The starting point of our description of the neutral $B \rightarrow \pi K$ system is the following isospin relation:

$$
\sqrt{2} A\left(B_{d}^{0} \rightarrow \pi^{0} K^{0}\right)+A\left(B_{d}^{0} \rightarrow \pi^{-} K^{+}\right)=-\left[(T+C)+P_{\mathrm{ew}}\right] \equiv 3 A_{3 / 2},
$$

where the combination $(T+C)$ originates from colour-allowed and colour-suppressed $\bar{b} \rightarrow$ $\bar{u} u \bar{s}$ tree-diagram-like topologies, $P_{\text {ew }}$ is due to electroweak penguin constributions, and $A_{3 / 2}$ reminds us that there is only an $I=3 / 2$ isospin component present in (4). Within the Standard Model, these amplitudes can be parametrized as follows:

$$
T+C=|T+C| e^{i \delta_{T+C}} e^{i \gamma}, \quad P_{\mathrm{ew}}=-\left|P_{\mathrm{ew}}\right| e^{i \delta_{\mathrm{ew}}},
$$

where $\delta_{T+C}$ and $\delta_{\text {ew }}$ denote CP-conserving strong phases. For the following considerations, we have to parametrize the $B_{d}^{0} \rightarrow \pi^{0} K^{0}$ decay amplitude in an appropriate way. If we make use of the unitarity of the CKM matrix and employ the Wolfenstein parametrization [13], generalized to include non-leading terms in $\lambda \equiv\left|V_{u s}\right|=0.22$ [14], we obtain

$$
\sqrt{2} A\left(B_{d}^{0} \rightarrow \pi^{0} K^{0}\right) \equiv P_{\mathrm{n}}=-\left(1-\frac{\lambda^{2}}{2}\right) \lambda^{2} A\left[1+\rho_{\mathrm{n}} e^{i \theta_{\mathrm{n}}} e^{i \gamma}\right] \mathcal{P}_{t c}^{\mathrm{n}}
$$

where $\rho_{\mathrm{n}} e^{i \theta_{\mathrm{n}}}$ takes the form

$$
\rho_{\mathrm{n}} e^{i \theta_{\mathrm{n}}}=\frac{\lambda^{2} R_{b}}{1-\lambda^{2}}\left[1-\left(\frac{\mathcal{P}_{u c}^{\mathrm{n}}-\mathcal{C}}{\mathcal{P}_{t c}^{\mathrm{n}}}\right)\right] .
$$

Here $\mathcal{P}_{t c}^{\mathrm{n}} \equiv\left|\mathcal{P}_{t c}^{\mathrm{n}}\right| e^{i \delta_{t c}^{\mathrm{n}}}$ and $\mathcal{P}_{u c}^{\mathrm{n}}$ correspond to differences of penguin topologies with internal top and charm and up and charm quarks, respectively. The amplitude $\mathcal{C}$ is due to insertions of 
current-current operators into colour-suppressed tree-diagram-like topologies, and

$$
A \equiv \frac{1}{\lambda^{2}}\left|V_{c b}\right|=0.81 \pm 0.06, \quad R_{b} \equiv \frac{1}{\lambda}\left(1-\frac{\lambda^{2}}{2}\right)\left|\frac{V_{u b}}{V_{c b}}\right|=\sqrt{\bar{\varrho}^{2}+\bar{\eta}^{2}}=0.41 \pm 0.07
$$

are the usual CKM factors. In order to parametrize the observable $R_{\mathrm{n}}$ defined in (3), it is useful to introduce the following quantities:

$$
r_{\mathrm{n}} \equiv \frac{|T+C|}{\sqrt{\left\langle\left|P_{\mathrm{n}}\right|^{2}\right\rangle}}, \quad \delta_{\mathrm{n}} \equiv \delta_{T+C}-\delta_{t c}^{\mathrm{n}},
$$

where

$$
\left\langle\left|P_{\mathrm{n}}\right|^{2}\right\rangle \equiv \frac{1}{2}\left(\left|P_{\mathrm{n}}\right|^{2}+\left|\overline{P_{\mathrm{n}}}\right|^{2}\right)
$$

is the CP-average of the $B_{d}^{0} \rightarrow \pi^{0} K^{0}$ decay amplitude specified in (6). Then we obtain [3, 15]:

$$
R_{\mathrm{n}}=1-\frac{2 r_{\mathrm{n}}}{u_{\mathrm{n}}}\left(h_{\mathrm{n}} \cos \delta_{\mathrm{n}}+k_{\mathrm{n}} \sin \delta_{\mathrm{n}}\right)+v^{2} r_{\mathrm{n}}^{2},
$$

where

$$
\begin{aligned}
& h_{\mathrm{n}}=\cos \gamma+\rho_{\mathrm{n}} \cos \theta_{\mathrm{n}}-q\left[\cos \omega+\rho_{\mathrm{n}} \cos \left(\theta_{\mathrm{n}}-\omega\right) \cos \gamma\right] \\
& k_{\mathrm{n}}=\rho_{\mathrm{n}} \sin \theta_{\mathrm{n}}+q\left[\sin \omega-\rho_{\mathrm{n}} \sin \left(\theta_{\mathrm{n}}-\omega\right) \cos \gamma\right],
\end{aligned}
$$

and

$$
\begin{gathered}
u_{\mathrm{n}}=\sqrt{1+2 \rho_{\mathrm{n}} \cos \theta_{\mathrm{n}} \cos \gamma+\rho_{\mathrm{n}}^{2}} \\
v=\sqrt{1-2 q \cos \omega \cos \gamma+q^{2}} .
\end{gathered}
$$

Moreover, we have introduced the electroweak penguin parameter

$$
q e^{i \omega} \equiv\left|\frac{P_{\mathrm{ew}}}{T+C}\right| e^{i\left(\delta_{\mathrm{ew}}-\delta_{T+C}\right)},
$$

which can be fixed theoretically [2] (see also [16]). This interesting observation was made by Neubert and Rosner in the context of the charged $B \rightarrow \pi K$ system. However, as (田) is also satisfied by the corresponding charged combination, the same feature can be used in the neutral strategy as well [3]. To this end, two electroweak penguin operators with tiny Wilson coefficients are neglected, as well as electroweak penguins with internal up and charm quarks. Furthermore, appropriate Fierz transformations of the remaining electroweak penguin operators are performed, and the $S U(3)$ flavour symmetry of strong interactions is applied. Finally, one arrives at the following result [2]:

$$
q e^{i \omega}=0.63 \times\left[\frac{0.41}{R_{b}}\right],
$$


where also factorizable $S U(3)$-breaking corrections have been taken into account. The amplitude $T+C$, i.e. the parameter $r_{\mathrm{n}}$, can be determined with the help of the decay $B^{+} \rightarrow \pi^{+} \pi^{0}$ by using the $S U(3)$ flavour symmetry of strong interactions [17]:

$$
T+C=-\sqrt{2} \frac{V_{u s}}{V_{u d}} \frac{f_{K}}{f_{\pi}} A\left(B^{+} \rightarrow \pi^{+} \pi^{0}\right) .
$$

Here the ratio $f_{K} / f_{\pi}=1.2$ of the kaon and pion decay constants takes into account factorizable $S U(3)$-breaking corrections. Electroweak penguin corrections to this expression can be taken into account theoretically [3, 18], but play a minor role in this case. The CLEO collaboration sees already some indication for $B^{ \pm} \rightarrow \pi^{ \pm} \pi^{0}$ modes [4], with a CP-averaged branching ratio of

$$
\mathrm{BR}\left(B^{ \pm} \rightarrow \pi^{ \pm} \pi^{0}\right)=\left(5.6_{-2.3}^{+2.6} \pm 1.7\right) \times 10^{-6}
$$

However, the statistical significance of the signal yield is not yet sufficient to claim an observation of this channel. Using nevertheless (19), and taking into account the measured $\mathrm{CP}$-averaged $B_{d} \rightarrow \pi^{0} K$ branching ratio, the combination of (9) and (18) yields

$$
r_{\mathrm{n}}=0.17 \pm 0.06
$$

where we have added the experimental errors in quadrature.

The bounds on $\gamma$ implied by $R_{\mathrm{n}}$ are related to extremal values of this observable. If we keep $r_{\mathrm{n}}$ and $\delta_{\mathrm{n}}$ as free parameters, we obtain the following minimal value for $R_{\mathrm{n}}$ [15]:

$$
\left.R_{\mathrm{n}}^{\min }\right|_{r_{\mathrm{n}}, \delta_{\mathrm{n}}}=\left[\frac{1+2 q \rho_{\mathrm{n}} \cos \left(\theta_{\mathrm{n}}+\omega\right)+q^{2} \rho_{\mathrm{n}}^{2}}{\left(1-2 q \cos \omega \cos \gamma+q^{2}\right)\left(1+2 \rho_{\mathrm{n}} \cos \theta_{\mathrm{n}} \cos \gamma+\rho_{\mathrm{n}}^{2}\right)}\right] \sin ^{2} \gamma
$$

On the other hand, if only the strong phase $\delta_{\mathrm{n}}$ is kept as an unknown quantity, $R_{\mathrm{n}}$ takes minimal and maximal values, which are given by [3]

$$
\left.R_{\mathrm{n}}^{\mathrm{ext}}\right|_{\delta_{\mathrm{n}}}=1 \pm 2 \frac{r_{\mathrm{n}}}{u_{\mathrm{n}}} \sqrt{h_{\mathrm{n}}^{2}+k_{\mathrm{n}}^{2}}+v^{2} r_{\mathrm{n}}^{2}
$$

Expressions (21) and (22) are the main equations of our paper. The parameter $\rho_{\mathrm{n}}$ is usually expected at the level of a few percent [19], and governs also direct CP violation in $B_{d} \rightarrow \pi^{0} K$; model calculations of the corresponding $\mathrm{CP}$ asymmetry give results within the range $[0.4 \%, 5 \%]$ [20]. However, it should be kept in mind that $\rho_{\mathrm{n}}$ may be enhanced by final-state-interaction processes [5]. These issues will be discussed in more detail in the following section.

The formulae given above apply also to the charged $B \rightarrow \pi K$ system, if we perform the following replacements:

$$
r_{\mathrm{n}} \rightarrow r_{\mathrm{c}} \equiv \frac{|T+C|}{\sqrt{\left\langle|P|^{2}\right\rangle}}, \quad \rho_{\mathrm{n}} e^{i \theta_{\mathrm{n}}} \rightarrow \rho e^{i \theta}, \quad \delta_{\mathrm{n}} \rightarrow \delta_{\mathrm{c}} \equiv \delta_{T+C}-\delta_{t c}^{\mathrm{c}}
$$

where

$$
P \equiv A\left(B^{+} \rightarrow \pi^{+} K^{0}\right)=-\left(1-\frac{\lambda^{2}}{2}\right) \lambda^{2} A\left[1+\rho e^{i \theta} e^{i \gamma}\right]\left|\mathcal{P}_{t c}^{\mathrm{c}}\right| e^{i \delta_{t c}^{c}}
$$


with

$$
\rho e^{i \theta}=\frac{\lambda^{2} R_{b}}{1-\lambda^{2}}\left[1-\left(\frac{\mathcal{P}_{u c}^{\mathrm{c}}+\mathcal{A}}{\mathcal{P}_{t c}^{\mathrm{c}}}\right)\right] .
$$

Here the amplitude $\mathcal{A}$ is due to annihilation topologies. Using (18), (19) and the measured $\mathrm{CP}$-averaged $B^{ \pm} \rightarrow \pi^{ \pm} K$ branching ratio, we obtain

$$
r_{\mathrm{c}}=0.21 \pm 0.06
$$

where we have again added the experimental errors in quadrature.

The parameter $\rho$ is a measure of the importance of certain rescattering effects [5]-[7], and can be probed by comparing $B^{ \pm} \rightarrow \pi^{ \pm} K$ with its $U$-spin counterpart $B^{ \pm} \rightarrow K^{ \pm} K$ [6, 6, 15]. To this end, we consider the following quantity

$$
K \equiv\left[\frac{1}{\epsilon R_{S U(3)}^{2}}\right]\left[\frac{\mathrm{BR}\left(B^{ \pm} \rightarrow \pi^{ \pm} K\right)}{\mathrm{BR}\left(B^{ \pm} \rightarrow K^{ \pm} K\right)}\right]=\frac{1+2 \rho \cos \theta \cos \gamma+\rho^{2}}{\epsilon^{2}-2 \epsilon \rho \cos \theta \cos \gamma+\rho^{2}},
$$

where $\epsilon \equiv \lambda^{2} /\left(1-\lambda^{2}\right)$, and

$$
R_{S U(3)}=\frac{F_{B \pi}\left(M_{K}^{2} ; 0^{+}\right)}{F_{B K}\left(M_{K}^{2} ; 0^{+}\right)}
$$

describes factorizable $U$-spin-breaking corrections. If we use the model of Bauer, Stech and Wirbel [21] to estimate the relevant form factors, we obtain $R_{S U(3)}=\mathcal{O}(0.7)$. The expression on the right-hand side of (27) implies the following allowed range for $\rho$ (for a detailed discussion, see [9] and [22]):

$$
\frac{1-\epsilon \sqrt{K}}{1+\sqrt{K}} \leq \rho \leq \frac{1+\epsilon \sqrt{K}}{|1-\sqrt{K}|}
$$

The present CLEO data give $\mathrm{BR}\left(B^{ \pm} \rightarrow K^{ \pm} K\right) / \mathrm{BR}\left(B^{ \pm} \rightarrow \pi^{ \pm} K\right)<0.3$ at $90 \%$ C.L. 幽. Consequently, using (29), this upper bound implies $\rho<0.15$ for $R_{S U(3)}=0.7$, and is not in favour of dramatic rescattering effects, although the upper bound is still one order of magnitude above the usual model calculations, making use of arguments based on factorization.

Let us finally note that the formalism discussed in this section can also be applied to the "mixed" $B_{d} \rightarrow \pi^{\mp} K^{ \pm}, B^{ \pm} \rightarrow \pi^{ \pm} K$ system. To this end, we have just to make appropriate replacements of variables, involving certain amplitudes $T$ and $P_{\mathrm{ew}}^{\mathrm{C}}$, which measure colourallowed tree-diagram-like and colour-suppressed electroweak penguin topologies, respectively. In order to fix $T$, arguments based on the factorization hypothesis have to be employed, and usually it is assumed that the colour-suppressed electroweak penguin amplitude $P_{\mathrm{ew}}^{\mathrm{C}}$ plays a very minor role. However, in contrast to (5), these quantities may be affected by rescattering processes. An interesting approach, making use of a heavy-quark expansion for non-leptonic $B$ decays, was recently proposed in Ref. [23], which could help to reduce the uncertainties related to $T$ and $P_{\mathrm{ew}}^{\mathrm{C}}$. It should also be useful to reduce the theoretical uncertainties of $r_{\mathrm{n}}$, $r_{\mathrm{c}}$ and $q e^{i \omega}$, which are due to non-factorizable $S U(3)$-breaking corrections. Moreover, this approach allows also a calculation of the parameters $\rho_{\mathrm{n}} e^{i \theta_{\mathrm{n}}}$ and $\rho e^{i \theta}$. We will not consider the $B_{d} \rightarrow \pi^{\mp} K^{ \pm}, B^{ \pm} \rightarrow \pi^{ \pm} K$ system further in this paper, and refer the reader to Refs. [3, 15], where detailed discussions can be found. Recently, also the utility of $B_{s} \rightarrow \pi K$ decays in this context was pointed out [24]. 


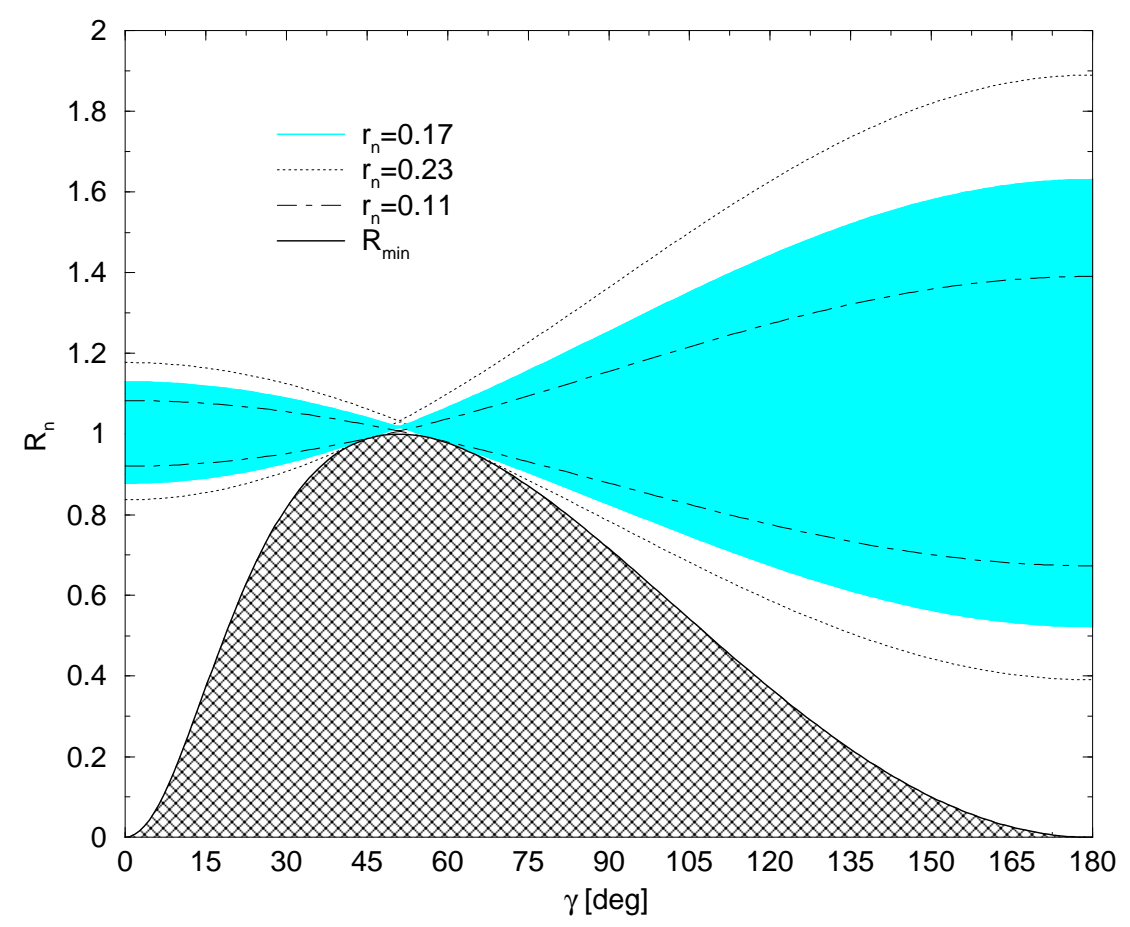

Figure 1: The dependence of the extremal values of $R_{\mathrm{n}}$ (neutral $B \rightarrow \pi K$ system) described by (21) and (22) on the CKM angle $\gamma$ for $q e^{i \omega}=0.63$ and $\rho_{\mathrm{n}}=0$.

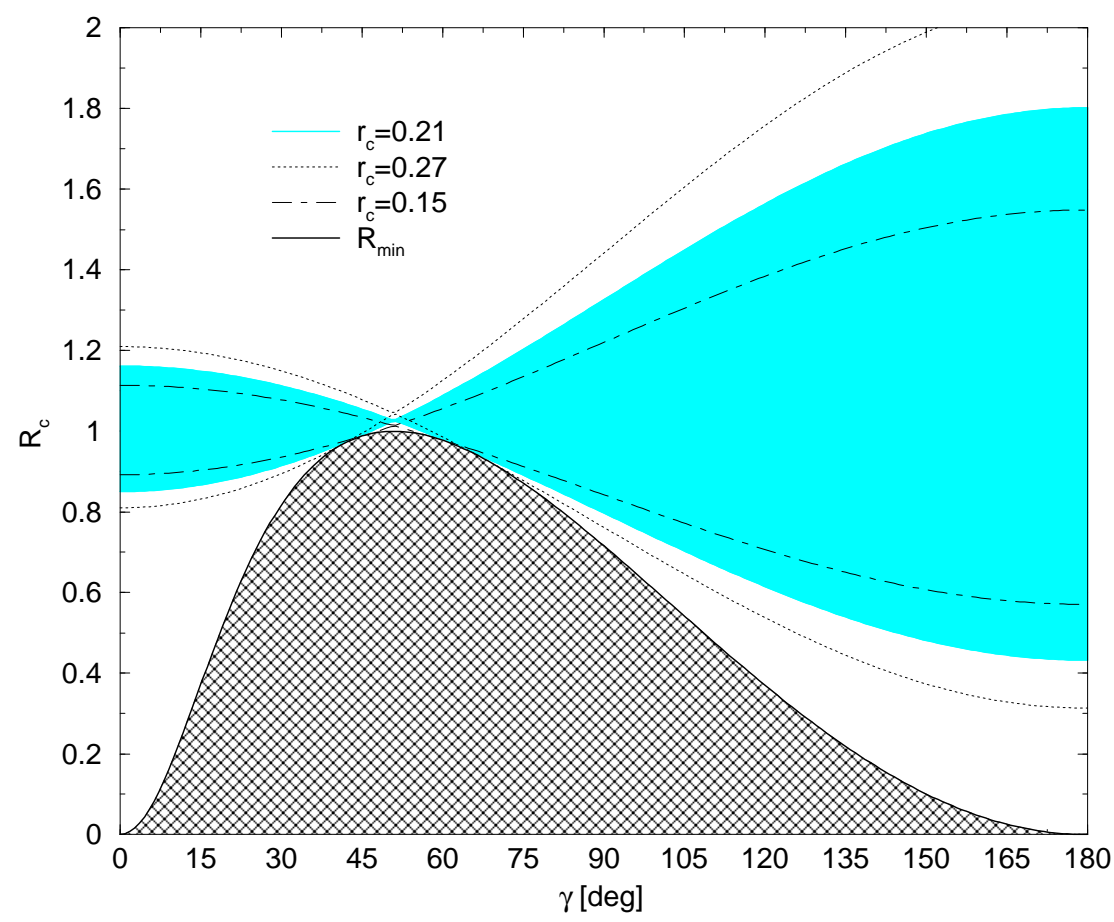

Figure 2: The dependence of the extremal values of $R_{\mathrm{c}}$ (charged $B \rightarrow \pi K$ system) corresponding to (21) and (22) on the CKM angle $\gamma$ for $q e^{i \omega}=0.63$ and $\rho=0$. 


\section{Bounds on $\gamma$ and Constraints in the $\bar{\varrho}-\bar{\eta}$ Plane}

The bounds on the CKM angle $\gamma$ implied by the CP-averaged branching ratios of the neutral $B \rightarrow \pi K$ decays are related to the extremal values of $R_{\mathrm{n}}$ given in (21) and (22). In Fig. 1, we show their dependence on $\gamma$ for $q e^{i \omega}=0.63$ and $\rho_{\mathrm{n}}=0 .{ }^{1}$ Here all values of $R_{\mathrm{n}}$ below the $R_{\min }$ curve are excluded. If $r_{\mathrm{n}}$ is fixed, for example to be equal to 0.17 , all values of $R_{\mathrm{n}}$ outside the shaded region are excluded, which is enlarged (reduced) for larger (smaller) values of $r_{\mathrm{n}}$. Fig. 1 allows us to read off immediately the allowed range for $\gamma$ corresponding to a given value of $R_{\mathrm{n}}$. Let us consider, for example, the central value of (3), $R_{\mathrm{n}}=0.6$. In this case, the $R_{\text {min }}$ curve implies the allowed range $0^{\circ} \leq \gamma \leq 21^{\circ} \vee 100^{\circ} \leq \gamma \leq 180^{\circ}$. If we use additional information on the parameter $r_{\mathrm{n}}$, we may put even stronger constraints on $\gamma$. For $r_{\mathrm{n}}=0.17$, we obtain, for instance, the allowed range $138^{\circ} \leq \gamma \leq 180^{\circ}$.

In the case of the charged $B \rightarrow \pi K$ system, bounds on $\gamma$ can be obtained in an analogous manner. The corresponding curves for the extremal values of $R_{\mathrm{c}}$ are shown in Fig. 2. There is some kind of complementarity between the neutral and charged $B \rightarrow \pi K$ systems, since the CLEO data favour $R_{\mathrm{n}}<1$ and $R_{\mathrm{c}}>1$. Consequently, we have to fix $r_{\mathrm{c}}$ in order to constrain $\gamma$ through the charged $B \rightarrow \pi K$ decays. For the central values of (2) and (26), $R_{\mathrm{c}}=1.3$ and $r_{\mathrm{c}}=0.21$, we obtain $87^{\circ} \leq \gamma \leq 180^{\circ}$.

The allowed ranges for $\gamma$ arising in the examples given above would be of particular phenomenological interest, as they would be complementary to the range of $\gamma$ arising from the usual indirect fits of the unitarity triangle [10. The most recent analysis [11] gives

$$
38^{\circ} \leq \gamma \leq 81^{\circ}
$$

In our examples of the bounds from the neutral $B \rightarrow \pi K$ system, there would be no overlap between these ranges, which could be interpreted as a manifestation of new physics [25, 26]. In particular, the second quadrant for $\gamma$ is favoured; other arguments for $\cos \gamma<0$ using $B \rightarrow P P, P V$ and $V V$ decays were recently given in [12] (see also [9]). However, the present data do not yet allow us to draw any definite conclusions. Before we can speculate on physics beyond the Standard Model, it is of course crucial to explore hadronic uncertainties. For the formalism used in this paper, this was done in [3]; within a different framework, similar considerations were also made for the charged and "mixed" $B \rightarrow \pi K$ systems in [27].

The theoretical accuracy of the bounds on $\gamma$ discussed in this section is limited both by non-factorizable $S U(3)$-breaking corrections and by rescattering processes. The former may affect the determination of the parameters $q e^{i \omega}$ and $r_{\mathrm{n}, \mathrm{c}}$, whereas the latter may lead to sizeable values of $\rho_{\mathrm{n}}$ and $\rho$. In order to control the non-factorizable $S U(3)$-breaking corrections, the "QCD factorization" approach presented in [23] appears to be very promising.

In the case of the neutral strategy, the parameter $\rho_{\mathrm{n}} e^{i \theta_{\mathrm{n}}}$ can be probed - and even taken into account in the bounds on $\gamma$ in an exact manner - through CP-violating effects. To this end, we consider the $B_{d} \rightarrow \pi^{0} K$ modes and require that the kaon be observed as a $K_{\mathrm{S}}$. The resulting final state is then an eigenstate of the $\mathrm{CP}$ operator with eigenvalue -1 , and we obtain the following time-dependent $\mathrm{CP}$ asymmetry [3]:

$$
a_{\mathrm{CP}}\left(B_{d}(t) \rightarrow \pi^{0} K_{\mathrm{S}}\right) \equiv \frac{\mathrm{BR}\left(B_{d}^{0}(t) \rightarrow \pi^{0} K_{\mathrm{S}}\right)-\mathrm{BR}\left(\overline{B_{d}^{0}}(t) \rightarrow \pi^{0} K_{\mathrm{S}}\right)}{\mathrm{BR}\left(B_{d}^{0}(t) \rightarrow \pi^{0} K_{\mathrm{S}}\right)+\mathrm{BR}\left(\overline{B_{d}^{0}}(t) \rightarrow \pi^{0} K_{\mathrm{S}}\right)}
$$

\footnotetext{
${ }^{1}$ In Fig. 11, we have assumed $0^{\circ} \leq \gamma \leq 180^{\circ}$, as implied by the measured CP-violating parameter $\varepsilon_{K}$ of the neutral kaon system.
} 


$$
=\mathcal{A}_{\mathrm{CP}}^{\mathrm{dir}}\left(B_{d} \rightarrow \pi^{0} K_{\mathrm{S}}\right) \cos \left(\Delta M_{d} t\right)+\mathcal{A}_{\mathrm{CP}}^{\mathrm{mix}}\left(B_{d} \rightarrow \pi^{0} K_{\mathrm{S}}\right) \sin \left(\Delta M_{d} t\right),
$$

where $\mathcal{A}_{\mathrm{CP}}^{\mathrm{dir}}\left(B_{d} \rightarrow \pi^{0} K_{\mathrm{S}}\right)$ and $\mathcal{A}_{\mathrm{CP}}^{\mathrm{mix}}\left(B_{d} \rightarrow \pi^{0} K_{\mathrm{S}}\right)$ are due to "direct" and "mixing-induced" $\mathrm{CP}$ violation, respectively. Using (6), these observables take the following form:

$$
\begin{gathered}
\mathcal{A}_{\mathrm{CP}}^{\mathrm{dir}}\left(B_{d} \rightarrow \pi^{0} K_{\mathrm{S}}\right)=-\frac{2 \rho_{\mathrm{n}} \sin \theta_{\mathrm{n}} \sin \gamma}{1+2 \rho_{\mathrm{n}} \cos \theta_{\mathrm{n}} \cos \gamma+\rho_{\mathrm{n}}^{2}} \\
\mathcal{A}_{\mathrm{CP}}^{\mathrm{mix}}\left(B_{d} \rightarrow \pi^{0} K_{\mathrm{S}}\right)= \\
-\left[\frac{\sin \left(\phi_{\mathrm{M}}^{(d)}+\phi_{K}\right)+2 \rho_{\mathrm{n}} \cos \theta_{\mathrm{n}} \sin \left(\phi_{\mathrm{M}}^{(d)}+\phi_{K}+\gamma\right)+\rho_{\mathrm{n}}^{2} \sin \left(\phi_{\mathrm{M}}^{(d)}+\phi_{K}+2 \gamma\right)}{1+2 \rho_{\mathrm{n}} \cos \theta_{\mathrm{n}} \cos \gamma+\rho_{\mathrm{n}}^{2}}\right] .
\end{gathered}
$$

The latter expression reduces to

$$
\mathcal{A}_{\mathrm{CP}}^{\mathrm{mix}}\left(B_{d} \rightarrow \pi^{0} K_{\mathrm{S}}\right)=-\sin \left(\phi_{\mathrm{M}}^{(d)}+\phi_{K}\right)=\mathcal{A}_{\mathrm{CP}}^{\mathrm{mix}}\left(B_{d} \rightarrow J / \psi K_{\mathrm{S}}\right)
$$

in the case of $\rho_{\mathrm{n}}=0$ [16]. Clearly, a violation of (34) and a sizeable value of the direct CP asymmetry (32) would signal that the parameter $\rho_{\mathrm{n}}$ cannot be neglected. Such a feature may either be due to large rescattering effects, or to new-physics contributions. The whole pattern of all experimentally observed $B \rightarrow \pi K$ and $B \rightarrow K \bar{K}$ decays may allow us to distinguish between these cases.

In the mixing-induced $\mathrm{CP}$ asymmetry (34), $\phi_{\mathrm{M}}^{(d)}=2 \arg \left(V_{t d}^{*} V_{t b}\right)$ is related to the weak $B_{d}^{0}-\overline{B_{d}^{0}}$ mixing phase, whereas $\phi_{K}$ is related to $K^{0}-\overline{K^{0}}$ mixing, and is negligibly small in the Standard Model. The combination $\phi_{d}=\phi_{\mathrm{M}}^{(d)}+\phi_{K}$ is equal to $2 \beta$ in the Standard Model, and can be determined "straightforwardly" through the "gold-plated" mode $B_{d} \rightarrow J / \psi K_{\mathrm{S}}$ at the $B$-factories. Strictly speaking, a measurement of $\mathcal{A}_{\mathrm{CP}}^{\text {mix }}\left(B_{d} \rightarrow J / \psi K_{\mathrm{S}}\right)$ allows us to determine only $\sin \phi_{d}$, i.e. to fix $\phi_{d}$ up to a twofold ambiguity. Several strategies were proposed in the literature to resolve this ambiguity [28].

If we assume that $\phi_{d}$ has been fixed this way, the observables (32) and (33) allow us to determine $\rho_{\mathrm{n}}$ and $\theta_{\mathrm{n}}$ as a function of $\gamma$. The general formulae given in the previous section allow us then to take into account these parameters in the curves shown in Fig. 1. The usual model calculations for non-leptonic $B$ decays give values for $\rho_{\mathrm{n}}$ at the level of a few percent. In order to illustrate the impact on the bounds on $\gamma$, let us take $\rho_{\mathrm{n}}=0.05$ and $\theta_{\mathrm{n}} \in\left\{0^{\circ}, 180^{\circ}\right\}$. For the example given above, we obtain then the allowed ranges $0^{\circ} \leq \gamma \leq\left(21^{\circ} \pm 1^{\circ}\right) \vee\left(100^{\circ} \pm 4^{\circ}\right) \leq$ $\gamma \leq 180^{\circ}$, and $\left(138^{\circ} \pm 2^{\circ}\right) \leq \gamma \leq 180^{\circ}$. The feature that the uncertainty due to $\rho_{\mathrm{n}}$ is larger in the case of $R_{\mathrm{n}}^{\min }$ can be understood easily by performing an expansion of (21) and (22) in powers of $\rho_{\mathrm{n}}$, and neglecting second-order terms of $\mathcal{O}\left(\rho_{\mathrm{n}}^{2}\right), \mathcal{O}\left(r_{\mathrm{n}} \rho_{\mathrm{n}}\right)$ and $\mathcal{O}\left(r_{\mathrm{n}}^{2}\right)$ :

$$
\begin{aligned}
\left.R_{\mathrm{n}}^{\min }\right|_{r_{\mathrm{n}}, \delta_{\mathrm{n}}} ^{\mathrm{L} . \mathrm{O}} & =\left[\frac{1+2 \rho_{\mathrm{n}} \cos \theta_{\mathrm{n}}(q-\cos \gamma)}{1-2 q \cos \gamma+q^{2}}\right] \sin ^{2} \gamma \\
\left.R_{\mathrm{n}}^{\text {ext }}\right|_{\delta_{\mathrm{n}}} ^{\mathrm{L} . \mathrm{O} .} & =1 \pm 2 r_{\mathrm{n}}|\cos \gamma-q| .
\end{aligned}
$$

Here we have moreover made use of (17), which gives $\omega=0$. Interestingly, as was noted for the charged $B \rightarrow \pi K$ system in [2], there are no terms of $\mathcal{O}\left(\rho_{\mathrm{n}}\right)$ present in (36), in contrast 


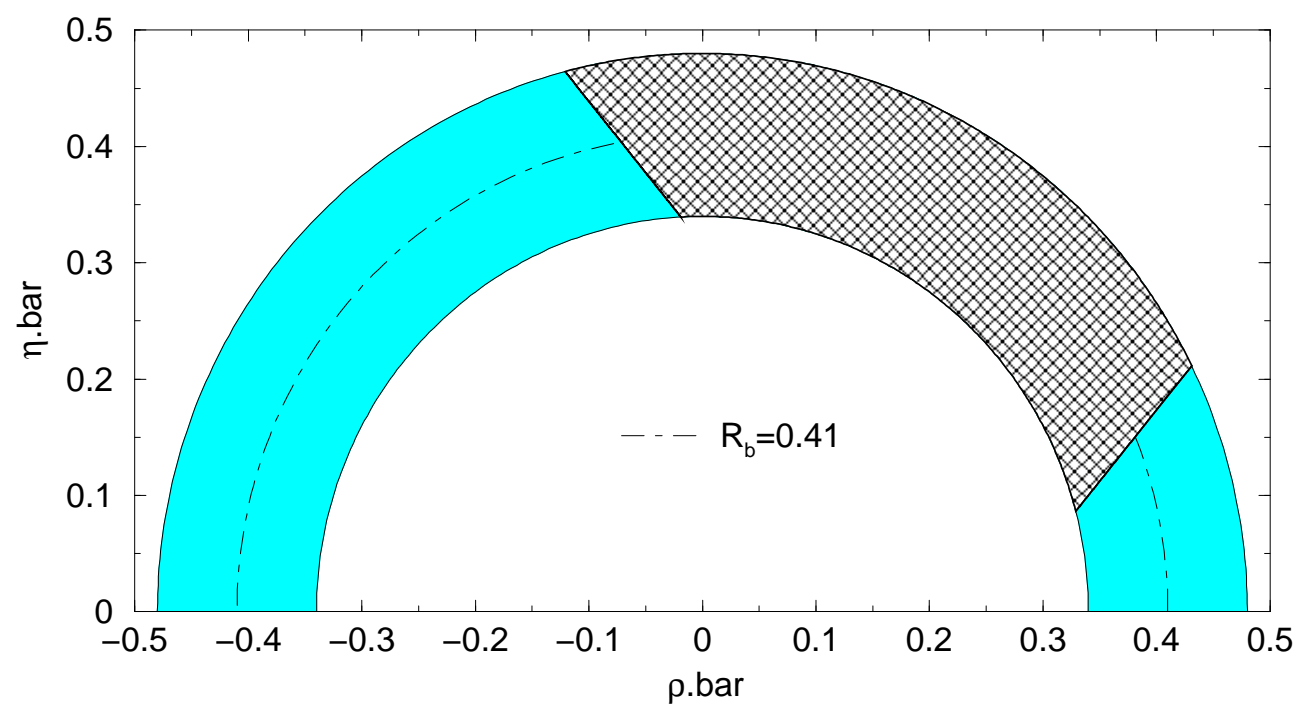

Figure 3: The constraints in the $\bar{\varrho}-\bar{\eta}$ plane implied by (21) for $R_{\mathrm{n}}=0.6, q e^{i \omega}=0.63 \times\left[0.41 / R_{b}\right]$, and $\rho_{\mathrm{n}}=0$. The shaded region is the allowed range for the apex of the unitarity triangle, whereas the "crossed" region is excluded through $\left.R_{\mathrm{n}}^{\min }\right|_{r_{\mathrm{n}}, \delta_{\mathrm{n}}}$ (see Fig. 1).

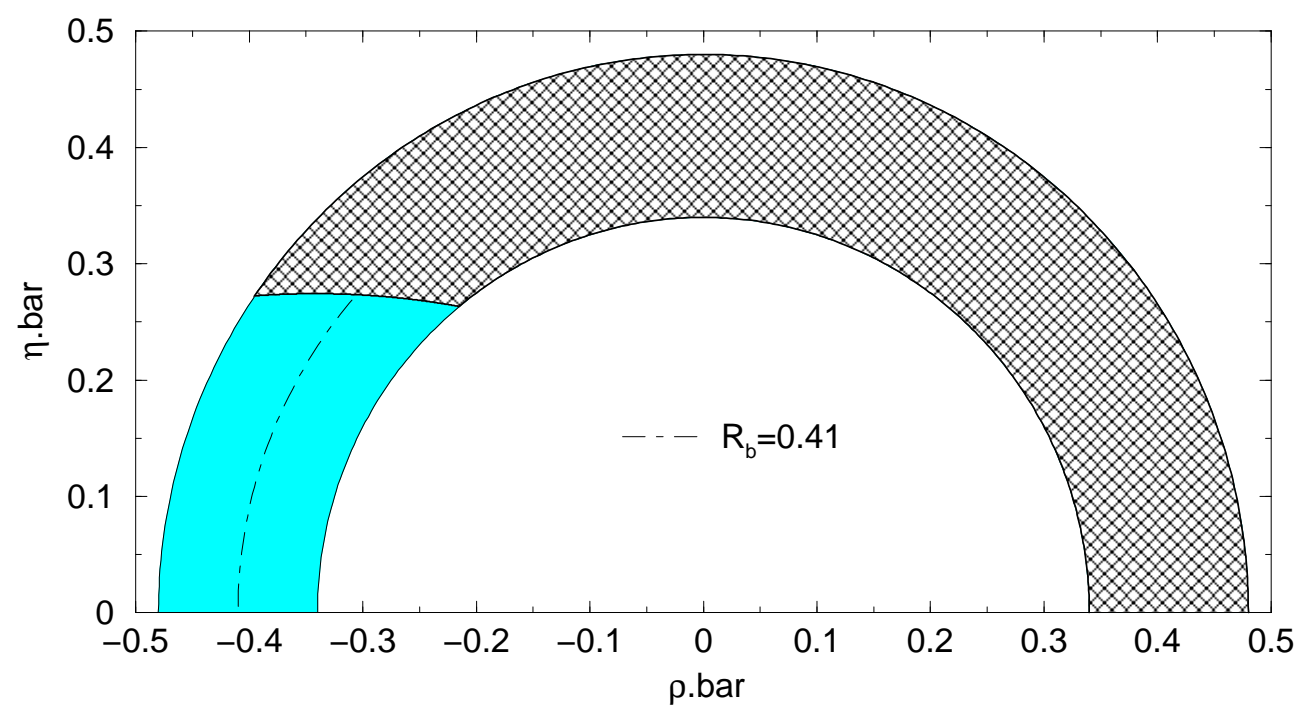

Figure 4: The constraints in the $\bar{\varrho}-\bar{\eta}$ plane implied by (22) for $R_{\mathrm{n}}=0.6, r_{\mathrm{n}}=0.17, q e^{i \omega}=$ $0.63 \times\left[0.41 / R_{b}\right]$, and $\rho_{\mathrm{n}}=0$. The shaded region is the allowed range for the apex of the unitarity triangle, whereas the "crossed" region is excluded through $\left.R_{\mathrm{n}}^{\text {ext }}\right|_{\delta_{\mathrm{n}}}$ (see Fig. 11). 
to (35). Consequently, the bounds on $\gamma$ related to (21) are affected more strongly by $\rho_{\mathrm{n}}$ then those implied by (22). In the case of the charged strategy, we have to use the $U$-spin flavour symmery, relating $B^{ \pm} \rightarrow \pi^{ \pm} K$ to $B^{ \pm} \rightarrow K^{ \pm} K$, in order to take into account the parameters $\rho$ and $\theta$ in the curves shown in Fig. 2 [3, 15]. To this end, the observable $K$ introduced in (27) has to be combined with the direct CP asymmetries in $B^{ \pm} \rightarrow \pi^{ \pm} K$ or $B^{ \pm} \rightarrow K^{ \pm} K$ modes.

In addition to the theoretical uncertainties associated with $S U(3)$-breaking and rescattering effects, another uncertainty of the constraints on $\gamma$ is due to the CKM factor $R_{b}$ in expression (17) for the electroweak penguin parameter $q e^{i \omega}$. Because of this feature, it is actually more appropriate to consider constraints in the $\bar{\varrho}-\bar{\eta}$ plane. A similar "trick" was also employed for $B_{d} \rightarrow \pi^{+} \pi^{-}$decays in [29], and recently for the charged $B \rightarrow \pi K$ system in [30].

The constraints in the $\bar{\varrho}-\bar{\eta}$ plane can be obtained straightforwardly from (21) and (22). In the former case, we obtain

$$
\cos \gamma=R_{\mathrm{n}} q \pm \sqrt{\left(1-R_{\mathrm{n}}\right)\left(1-R_{\mathrm{n}} q^{2}\right)}
$$

whereas we have in the latter case

$$
\cos \gamma=\frac{1-R_{\mathrm{n}} \pm 2 q r_{\mathrm{n}}+\left(1+q^{2}\right) r_{\mathrm{n}}^{2}}{2 r_{\mathrm{n}}\left(q r_{\mathrm{n}} \pm 1\right)}
$$

In these expressions, we have assumed, for simpliciy, $\rho_{\mathrm{n}}=0$ and $\omega=0$. For the charged $B \rightarrow \pi K$ system, we obtain analogous expressions. The right-hand sides of these formulae depend implicitly on the CKM factor $R_{b}$ through the electroweak penguin parameter $q e^{i \omega}$, which is given by (17). Consequently, it is actually more appropriate to consider contours in the $\bar{\varrho}-\bar{\eta}$ plane instead of the CKM angle $\gamma$. They can be obtained with the help of (37) and (38) by taking into account [14]

$$
\bar{\varrho}=R_{b} \cos \gamma, \quad \bar{\eta}=R_{b} \sin \gamma,
$$

and are illustrated in Figs. 3 and 4 for the examples given in the previous section.

\section{Bounds on Strong Phases}

If we use the general expression (11) for $R_{\mathrm{n}}$, we can determine $\cos \delta_{\mathrm{n}}$ as a function of $\gamma$ :

$$
\cos \delta_{\mathrm{n}}=\frac{1}{h_{\mathrm{n}}^{2}+k_{\mathrm{n}}^{2}}\left[\frac{\left(1-R_{\mathrm{n}}+v^{2} r_{\mathrm{n}}^{2}\right) u_{\mathrm{n}} h_{\mathrm{n}}}{2 r_{\mathrm{n}}} \pm k_{\mathrm{n}} \sqrt{h_{\mathrm{n}}^{2}+k_{\mathrm{n}}^{2}-\left[\frac{\left(1-R_{\mathrm{n}}+v^{2} r_{\mathrm{n}}^{2}\right) u_{\mathrm{n}}}{2 r_{\mathrm{n}}}\right]^{2}}\right]
$$

In Fig. 5, we show the dependence of $\cos \delta_{\mathrm{n}}$ for various values of $R_{\mathrm{n}}$ in the case of $q e^{i \omega}=0.63$ and $r_{\mathrm{n}}=0.17$. From this figure, also the allowed range for $\gamma$ can be read off for a given value of $R_{\mathrm{n}}$. For the central value $R_{\mathrm{n}}=0.6$ of the present CLEO data, we obtain moreover $-1 \leq \cos \delta_{\mathrm{n}} \leq-0.86$. Performing the replacements given in (23), (40) applies also to the charged $B \rightarrow \pi K$ system. The corresponding contours in the $\gamma-\cos \delta_{\mathrm{c}}$ plane are shown in Fig. 6. For $R_{\mathrm{c}}=1.3$, we obtain $+0.27 \leq \cos \delta_{\mathrm{c}} \leq+1$.

As can be seen in (9) and (23), we have $\delta_{\mathrm{n}}-\delta_{\mathrm{c}}=\delta_{t c}^{\mathrm{c}}-\delta_{t c}^{\mathrm{n}}$, where $\delta_{t c}^{\mathrm{c}}$ and $\delta_{t c}^{\mathrm{n}}$ denote the strong phases of the amplitudes $\mathcal{P}_{t c}^{c}$ and $\mathcal{P}_{t c}^{\mathrm{n}}$, which describe the differences of penguin 


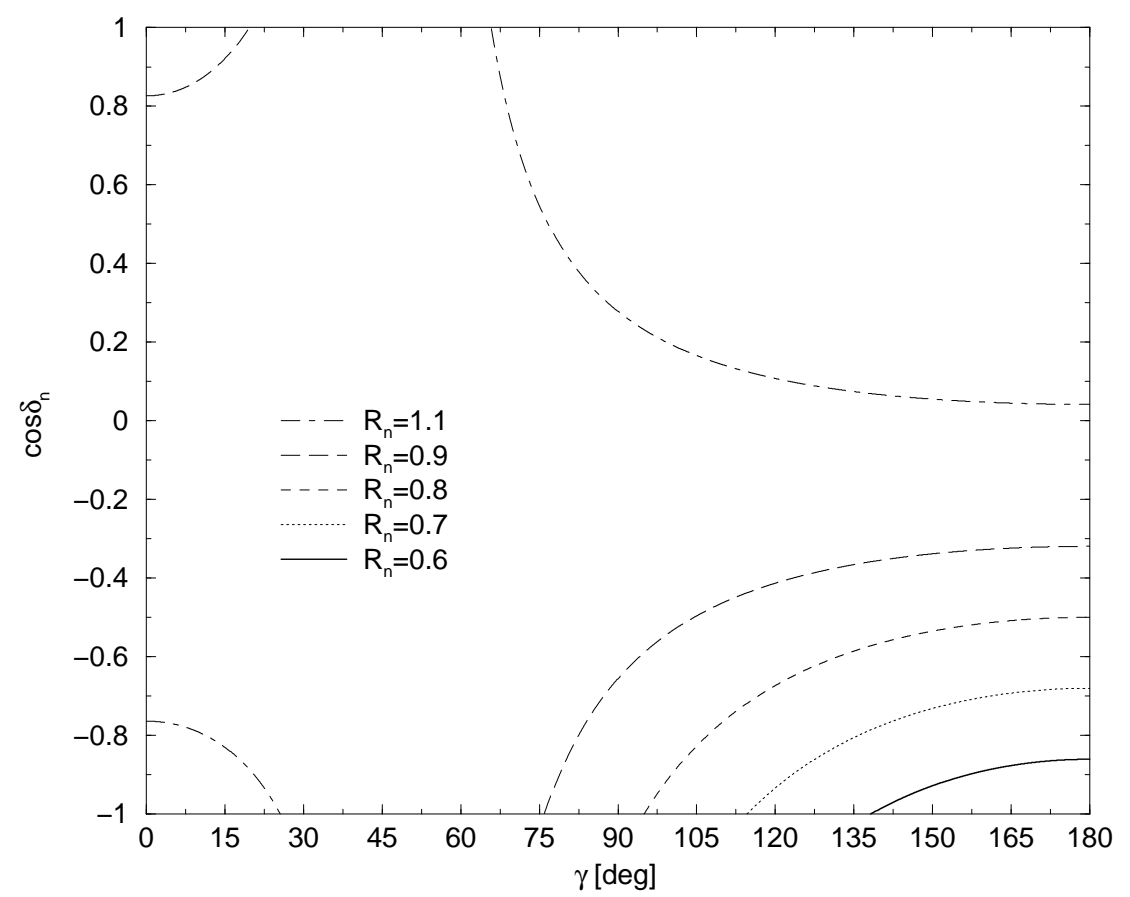

Figure 5: The dependence of $\cos \delta_{\mathrm{n}}$ on $\gamma$ for various values of $R_{\mathrm{n}}$ in the case of $q e^{i \omega}=0.63$ and $r_{\mathrm{n}}=0.17$. Rescattering effects are neglected, i.e. $\rho_{\mathrm{n}}=0$.

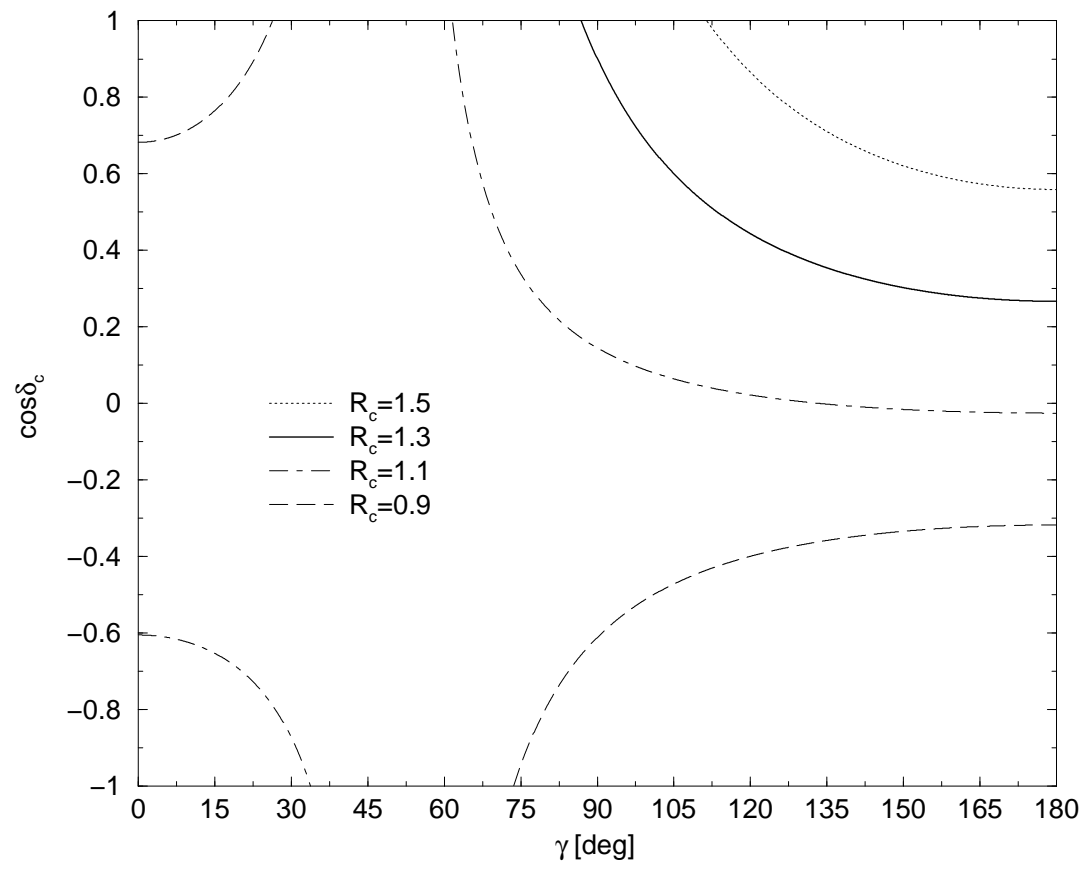

Figure 6: The dependence of $\cos \delta_{\mathrm{c}}$ on $\gamma$ for various values of $R_{\mathrm{c}}$ in the case of $q e^{i \omega}=0.63$ and $r_{\mathrm{c}}=0.21$. Rescattering effects are neglected, i.e. $\rho=0$. 


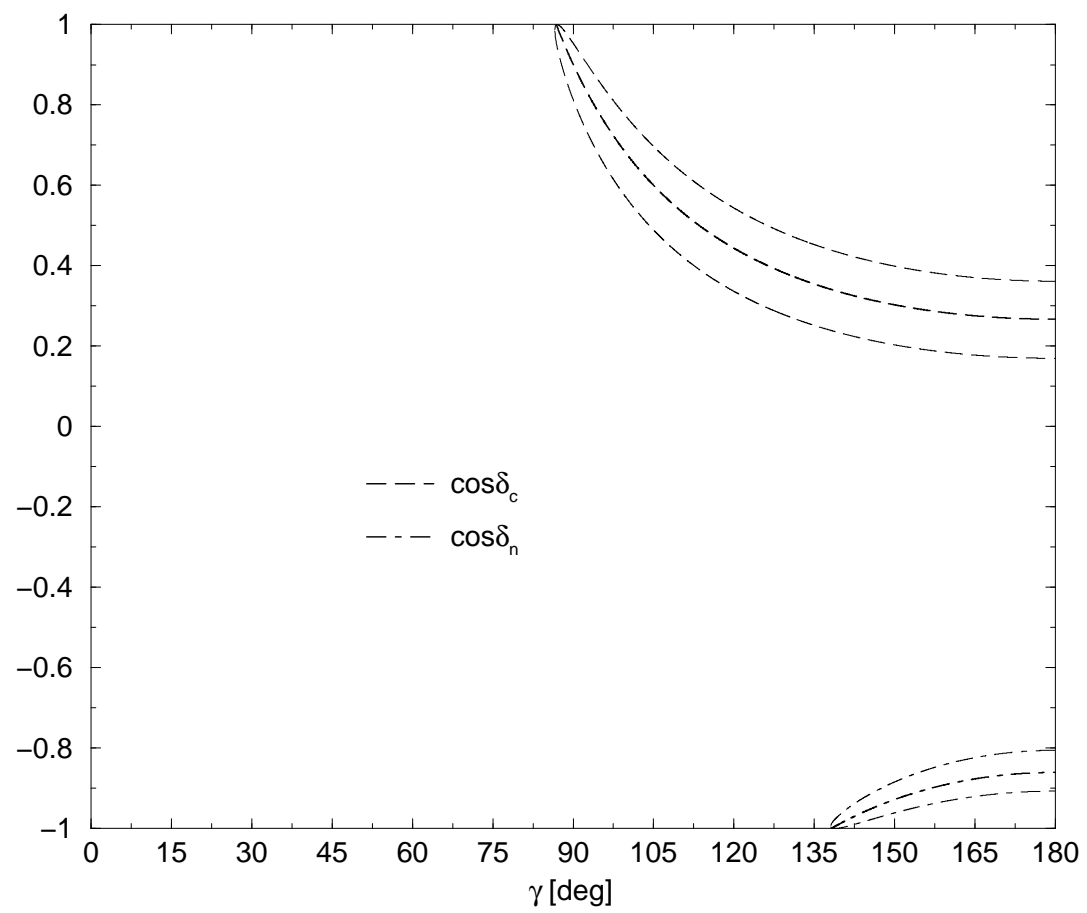

Figure 7: The dependence of $\cos \delta_{\mathrm{c}}$ and $\cos \delta_{\mathrm{n}}$ on $\gamma$ for $R_{\mathrm{c}}=1.3, r_{\mathrm{c}}=0.21, R_{\mathrm{n}}=0.6$, $r_{\mathrm{n}}=0.17, q e^{i \omega}=0.63$ in the presence of large rescattering effects (thin lines), corresponding to $\rho e^{i \theta}=\rho_{\mathrm{n}} e^{i \theta_{\mathrm{n}}}=0.1 \times \exp \left(i 90^{\circ}\right)$.

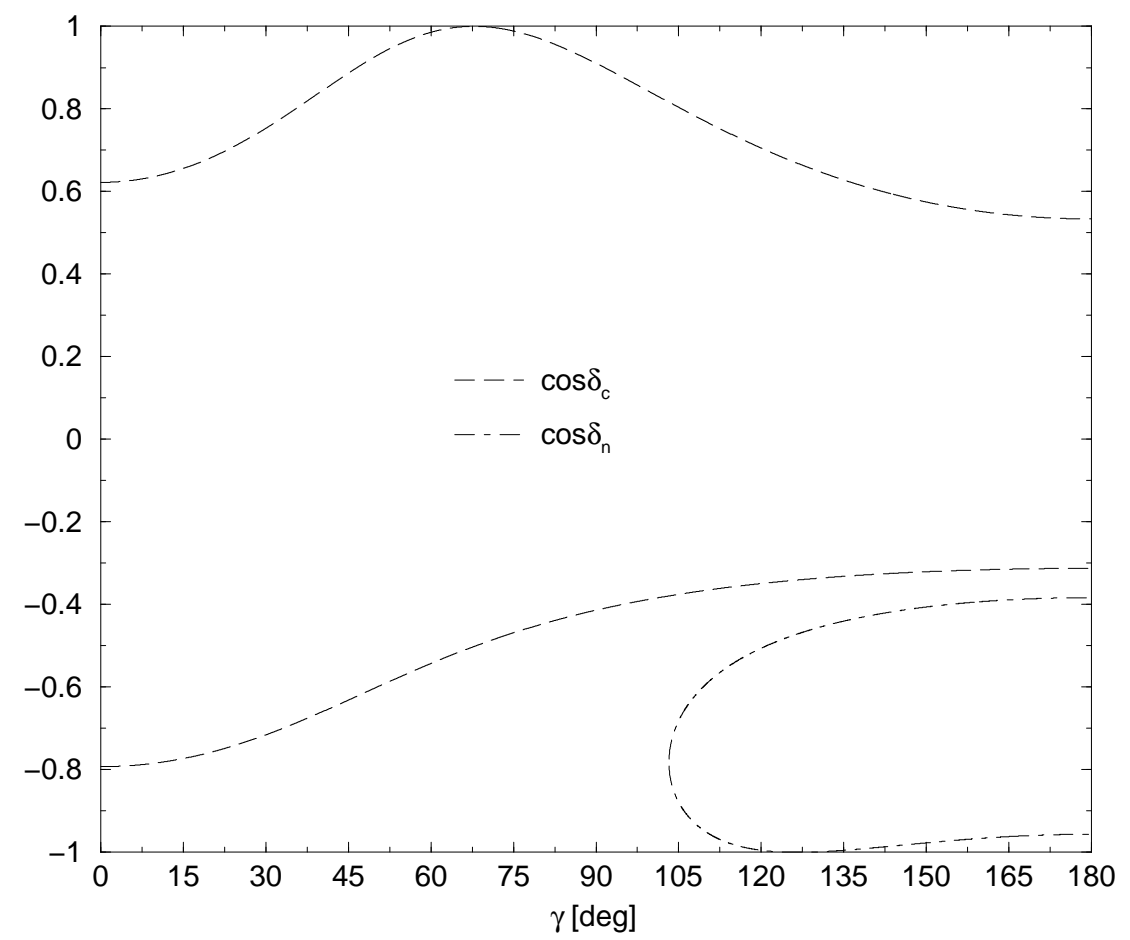

Figure 8: The dependence of $\cos \delta_{\mathrm{c}}$ and $\cos \delta_{\mathrm{n}}$ on $\gamma$ for $R_{\mathrm{c}}=1.3, r_{\mathrm{c}}=0.21, R_{\mathrm{n}}=0.6, r_{\mathrm{n}}=0.17$, $\rho=\rho_{\mathrm{n}}=0$ for a modified electroweak penguin parameter, given by $q e^{i \omega}=1.26 \times \exp \left(i 45^{\circ}\right)$. 
topologies with internal top- and charm-quark exchanges of the decays $B^{+} \rightarrow \pi^{+} K^{0}$ and $B_{d}^{0} \rightarrow \pi^{0} K^{0}$, respectively. These penguin topologies consist of QCD and electroweak penguins, where the latter contribute to $B^{+} \rightarrow \pi^{+} K^{0}$ only in colour-suppressed form. In contrast, $B_{d}^{0} \rightarrow$ $\pi^{0} K^{0}$ receives contributions both from colour-allowed and from colour-suppressed electroweak penguins. Nevertheless, they are expected to be at most of $\mathcal{O}(20 \%)$ of the $B_{d}^{0} \rightarrow \pi^{0} K^{0}$ QCD penguin amplitude. If we neglect the electroweak penguins and make use of isospin flavoursymmetry arguments, we obtain $\mathcal{P}_{t c}^{\mathrm{n}} \approx \mathcal{P}_{t c}^{\mathrm{c}}$, yielding $\delta_{\mathrm{n}} \approx \delta_{\mathrm{c}}$ and $\cos \delta_{\mathrm{n}} \approx \cos \delta_{\mathrm{c}}$. Employing moreover "factorization", these cosines are expected to be close to +1 .

Consequently, as the present CLEO data are in favour of $\cos \delta_{\mathrm{n}}<0$ and $\cos \delta_{\mathrm{c}}>0$, we arrive at a "puzzling" situation, although it is of course too early to draw definite conclusions. If future data should confirm this "discrepancy", it may be an indication for new-physics contributions to the electroweak penguin sector, or a manifestation of large non-factorizable $S U(3)$-breaking effects. Since the parameter $\rho_{\mathrm{n}}$ enters in expression (11) for $R_{\mathrm{n}}$ in the term proportional to $r_{\mathrm{n}}$, it can be regarded as a second-order effect and does not play a dramatic role for the contraints on $\cos \delta_{\mathrm{n}(\mathrm{c})}$ and $\gamma$. This feature is illustrated in Fig. 7 for the central values of the present CLEO data.

In Fig. 8, we consider the impact of a modified electroweak penguin parameter, $q e^{i \omega}=$ $1.26 \times \exp \left(i 45^{\circ}\right)$, which differs significantly from the $S U(3)$ Standard-Model expression (17). In this case, the discrepancy between $\cos \delta_{\mathrm{n}}$ and $\cos \delta_{\mathrm{c}}$ would be essentially resolved, favouring values of $\mathcal{O}(-0.5)$, which would still be in conflict with the factorization expectation. A value of $q e^{i \omega}=1.26 \times \exp \left(i 45^{\circ}\right)$ may be due to $\mathrm{CP}$-conserving new-physics contributions to the electroweak penguin sector [26]. In general, new physics will also lead to CP-violating contributions, which may lead to sizeable direct $\mathrm{CP}$ violation in $B_{d} \rightarrow \pi^{0} K_{\mathrm{S}}$, and to a violation of (34). Consequently, as we have already emphasized above, it would be an important task to measure the $\mathrm{CP}$-violating observables of this decay.

If the new-physics contributions are CP-conserving, it will be hard to distinguish them from large non-factorizable flavour-symmetry-breaking effects, which may also shift the parameter $q e^{i \omega}$ from (17). In Refs. [26, 27], it was argued that these effects are very small, whereas we gave a more critical picture in [3]. Also the approach proposed in [23] is in favour of small non-factorizable effects. The deviation of $q e^{i \omega}=1.26 \times \exp \left(i 45^{\circ}\right)$ used in the example given in Fig. 8 from (17) would probably be too large to be explained by $S U(3)$ breaking in a "natural" way. However, there may be additional sources for flavour-symmetry-breaking effects. An example is $\pi^{0}-\eta, \eta^{\prime}$ mixing, which has not yet been considered for $B \rightarrow \pi^{0} K$ decays. In a recent paper 31], it was emphasized that isospin violation arising from such effects could mock new physics in the extraction of the CKM angle $\alpha$ from $B \rightarrow \pi \pi$ isospin relations. It would be interesting to extend these studies also to the $B \rightarrow \pi K$ approaches to probe $\gamma$.

\section{Conclusions}

As we have pointed out in Ref. [3], the neutral $B \rightarrow \pi K$ strategy could be useful to constrain - and eventually determine $-\gamma$ in an analogous manner as the strategy of Neubert and Rosner [2] using charged $B \rightarrow \pi K$ modes. The most recent CLEO data look very interesting in this respect. As we have illustrated in Figs. 1 1 , improved measurements of both the neutral and the charged modes, in particular taken together, could give a powerful constraint on $\gamma$. 
There is some indication that the second quadrant for $\gamma$ is preferred. This is in contrast to the standard analysis of the unitarity triangle, which favours the first quadrant. Unfortunately, no definite conclusions can be drawn at present. This "discrepancy" between the $B \rightarrow \pi K$ approaches and the standard analysis of the unitarity triangle could turn out to be more pronounced when the $B$-decay data improve and the lower bound on $B_{s}^{0}-\overline{B_{s}^{0}}$ mixing will be raised, forcing the upper bound on $\gamma$ from the standard analysis to be even smaller than presently known.

We have also pointed out that the CLEO data suggest bounds on the strong phases $\delta_{\mathrm{n}}$ and $\delta_{\mathrm{c}}$ with $\cos \delta_{\mathrm{n}}<0$ and $\cos \delta_{\mathrm{c}}>0$. The substantial deviation of $\delta_{\mathrm{n}}$ from $\delta_{\mathrm{c}}$ and the negative value of $\cos \delta_{\mathrm{n}}$, if confirmed by improved data, would either indicate substantial new-physics contributions to the electroweak penguin sector, or large non-factorizable $S U(3)$ breaking effects. In order to distinguish between these possibilties, detailed studies of the various patterns of new-physics effects in all $B \rightarrow \pi K$ decays are essential, as well as critical analyses of possible sources for $S U(3)$ breaking. We hope that future studies following the strategies discussed in this paper will eventually shed light on the physics beyond the Standard Model.

This work has been supported in part by the German Bundesministerium für Bildung und Forschung under contract 05HT9WOA0. 


\section{References}

[1] R. Fleischer and T. Mannel, Phys. Rev. D57 (1998) 2752.

[2] M. Neubert and J.L. Rosner, Phys. Lett. B441 (1998) 403; Phys. Rev. Lett. 81 (1998) 5076 .

[3] A.J. Buras and R. Fleischer, Eur. Phys. J. C11 (1999) 93.

[4] CLEO Collaboration (D. Cronin-Hennessy et al.), CLEO 99-18 hep-ex/0001010].

[5] See, for instance, L. Wolfenstein, Phys. Rev. D52 (1995) 537; J. Donoghue, E. Golowich, A. Petrov and J. Soares, Phys. Rev. Lett. 77 (1996) 2178; B. Blok, M. Gronau and J. Rosner, Phys. Rev. Lett. 78 (1997) 3999; J.-M. Gérard and J. Weyers, Eur. Phys. J. C7 (1999) 1; M. Neubert, Phys. Lett. B424 (1998) 152; A. Falk, A. Kagan, Y. Nir and A. Petrov, Phys. Rev. D57 (1998) 4290; D. Atwood and A. Soni, Phys. Rev. D58 (1998) 036005 .

[6] A.J. Buras, R. Fleischer and T. Mannel, Nucl. Phys. B533 (1998) 3.

[7] A. Falk, A. Kagan, Y. Nir and A. Petrov, Phys. Rev. D57 (1998) 4290.

[8] CLEO Collaboration (S. Chen et al.), CLEO 99-17 [hep-ex/0001009].

[9] R. Fleischer, DESY 00-014 hep-ph/0001253, to appear in Eur. Phys. J. C.

[10] A. Ali and D. London, Eur. Phys. J. C9 (1999) 687; A.J. Buras, in the Proceedings of the Lake Louise Winter Institute: Electroweak Physics, Lake Louise, Alberta, Canada, 14-20 February 1999, eds. A. Astbury et al. (World Scientific, Singapore, 1999), p. 1 [hep-ph/9905437]; S. Schael, Phys. Rept. 313 (1999) 293.

[11] A. Ali and D. London, DESY 00-026 [hep-ph/0002167, to appear in the Proceedings of the 3rd Workshop on Physics and Detectors for DA $\Phi$ NE, Frascati, Italy, 16-19 November, 1999.

[12] W.-S. Hou and K.-C. Yang, Phys. Rev. D61 (2000) 073014; W.-S. Hou, J.G. Smith and F. Würthwein, NTU-HEP-99-25 [hep-ex/9910014].

[13] L. Wolfenstein, Phys. Rev. Lett. 51 (1983) 1945.

[14] A.J. Buras, M.E. Lautenbacher and G. Ostermaier, Phys. Rev. D50 (1994) 3433.

[15] R. Fleischer, Eur. Phys. J. C6 (1999) 451; Phys. Lett. B435 (1998) 221.

[16] R. Fleischer, Phys. Lett. B365 (1996) 399.

[17] M. Gronau, J.L. Rosner and D. London, Phys. Rev. Lett. 73 (1994) 21.

[18] M. Gronau, D. Pirjol and T.-M. Yan, Phys. Rev. D60 (1999) 034021. 
[19] M. Gronau, O.F. Hernández, D. London and J.L. Rosner, Phys. Rev. D52 (1995) 6356 and 6374 .

[20] A. Ali, G. Kramer and C.-D. Lü, Phys. Rev. D59 (1999) 014005.

[21] M. Bauer, B. Stech and M. Wirbel, Z. Phys. C29 (1985) 637 and C34 (1987) 103.

[22] D. Pirjol, Phys. Rev. D60 (1999) 054020.

[23] M. Beneke, G. Buchalla, M. Neubert and C.T. Sachrajda, Phys. Rev. Lett. 83 (1999) 1914.

[24] M. Gronau and J.L. Rosner, TECHNION-PH-00-25 hep-ph/0003119].

[25] For recent discussions, see D. Choudhury, B. Dutta and A. Kundu, Phys. Lett. B456 (1999) 185; X.-G. He, C.-L. Hsueh and J.-Q. Shi, Phys. Rev. Lett. 84 (2000) 18; R. Fleischer and J. Matias, Phys. Rev. D61 (2000) 74004.

[26] Y. Grossman, M. Neubert and A.L. Kagan, SLAC-PUB-8243 hep-ph/9909297.

[27] M. Neubert, J. High Energy Phys. 9902 (1999) 014.

[28] See, for example, Y. Grossman and H.R. Quinn, Phys. Rev. D56 (1997) 7259; J. Charles, A. Le Yaouanc, L. Oliver, O. Pène and J.-C. Raynal, Phys. Lett. B425 (1998) 375; A.S. Dighe, I. Dunietz and R. Fleischer, Phys. Lett. B433 (1998) 147; R. Fleischer, Phys. Rev. D60 (1999) 073008.

[29] J. Charles, Phys. Rev. D59 (1999) 054007.

[30] M. Neubert, CLNS 00/1660 [hep-ph/0001334, to appear in the Proceedings of the Trieste Summer School in Particle Physics (Part II), Trieste, Italy, 21 June - 9 July, 1999.

[31] S. Gardner, Phys. Rev. D59, 077502 (1999). 